\title{
Chronic nail biting, orthodontic treatment and Enterobacteriaceae in the oral cavity
}

\author{
Alagesan Chinnasamy ${ }^{1}$, Karthikeyan Ramalingam ${ }^{2}$, Pallu Chopra ${ }^{2}$, Vidhya Gopinath ${ }^{3}$, Gyan-Prakash Bishnoi ${ }^{2}$, \\ Gurveen Chawla ${ }^{2}$
}

${ }^{1}$ Population Oral Health, Melbourne Dental School, The University of Melbourne, Australia

${ }^{2}$ Oral Pathology and Microbiology, Surendera Dental College \& Research Institute, Rajasthan, India

${ }^{3}$ Saveetha Dental College, Chennai, India

Correspondence:

720 Swanston Street, Room 5.235

Royal Dental Hospital of Melbourne

The University of Melbourne

Melbourne 3053

Australia

alagesan2006@yahoo.co.uk

\begin{abstract}
Chinnasamy A, Ramalingam K, Chopra P, Gopinath V, Bishnoi GP, Chawla G. Chronic nail biting, orthodontic treatment and Enterobacteriaceae in the oral cavity. J Clin Exp Dent. 2019;11(12):e1157-62. http://www.medicinaoral.com/odo/volumenes/v11i12/jcedv11i12p1157.pdf
\end{abstract}

Received: 08/07/2019 Accepted: 04/11/2019

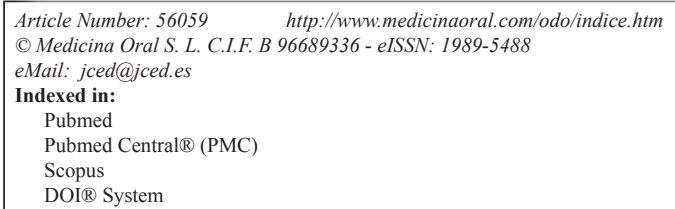

\begin{abstract}
Background: Chronic nail biting is common in children and young adults. Auto inoculation of environmental pathogens can manifest as infection in distant organs. Multi-drug resistance gram negative bacteria are on the rise globally. Several of the foodborne bacteria fall within the Enterobacteriaceae family but very few studies have explored these microbes in the oral cavity of children with chronic nail-biting habit or orthodontic treatment. The study aims to investigate oral load of Enterobacteriaceae in children with chronic nail-biting habit and or those undergoing orthodontic treatment

Material and Methods: 150 children (no nail-biting $n=30$, nail biting $n=60$, fixed orthodontic treatment $n=30$ and a combination of fixed orthodontic appliance use and nail-biting habit $\mathrm{n}=30$ ) were assessed for culture based microbiological investigation. The concentrated oral rinse technique was used. The rinse was inoculated in MacConkey's and Blood Agar. The gram stained culture was subjected to biochemical tests for sub-species identification using Biomerieux Vitek 2 Compact Automated Microbiological Analyzer. Fisher's exact and Kruskal Wallis with post hoc analysis using Dunn method was performed to test association and difference between groups.

Results: Enterobacteriaceae was positive for $72 \%$ of the children. Of them, nail biting or orthodontic treatment group comprised $89 \%$. Those with a combination of nail biting and undergoing orthodontic treatment exhibited highest CFU $/ \mathrm{ml}$ and those without nail biting or orthodontic treatment exhibited the lowest. Three of the four organisms isolated tested positive in the orthodontic treatment group. E. coli was positive in $38 \%$ of the children while Klebsiella and E. cloacae were isolated exclusively in the orthodontic treatment group.

Conclusions: Chronic nail biting or the use of fixed orthodontic appliances is associated with higher incidence of Enterobacteriaceae in the oral cavity. Oral health professionals play an important role in preventing multi drug resistance infectious diseases.
\end{abstract}

Key words: Enterobacteriaceae, nail-biting, Onychophagia, orthodontic treatment. 


\section{Introduction}

Onychophagia or nail biting is an oral compulsive behavioural disorder particularly common in children and young adults. Its prevalence is estimated to be between 6 to $45 \%$ (1-3). Although habitual nail biting is often considered to be harmless and wanes off as age advances, infrequently they have been associated with a range of psychological $(1,4)$, dental $(5-9)$, systemic infections and diseases $(10,11)$.

The oral cavity harbours a diverse set of more than 1,000 bacterial species (12). Nail biting increases the carriage of several opportunistic environmental microorganisms into the oral cavity. Among those include Enterobacteriaceae, a group of facultative anaerobic gram-negative bacteria that are thought to be transient or non-resident members of the oral cavity in healthy individuals (13). Some potentially pathogenic intestinal microorganisms of the Enterobacteriaceae family like Escherichia Coli (E. coli) and Enterobacter have been noted in the oral cavity of nail biters $(14,15)$.

Since most of the orthodontic treatment is carried out in school age children removal of food debris becomes challenging in difficult to reach sites in and around the appliances that are in close proximity to the oral tissues. This acts as a reservoir favouring microbial growth. With less than optimal hand hygiene compounded with the habit of nail biting, children can accidentally transport environmental pathogens to the oral cavity. Studies have identified children with the combination of nail biting and the use of orthodontic appliances exhibit significantly higher incidence of Enterobacteriaceae than those with nail biting alone $(16,17)$. Repetitive habitual nail biting compounded with less than optimal oral hygiene in children's receiving orthodontic treatment can carry the microbial loads to distant organs like the lungs, intestine and present as systemic manifestation altering the balance between health and disease (18-20). The test hypothesis is that chronic nail-biting habit or orthodontic treatment in isolation or in combination does not increase oral Enterobacteriaceae count. As such, this study aims to investigate oral load of Enterobacteriaceae in children with chronic nail-biting habit and or those undergoing orthodontic treatment compared to those with no nail biting or no orthodontic treatment.

\section{Material and Methods}

A culture based microbiological investigation was carried out among school children between six and 17 years attending the outpatient clinic at Surendara Dental College \& Research Institute, India. An Institutional Ethical Committee approval (SDC/IEC/2016/013) was obtained for this research work. Participating children and accompanying parents were informed of the study and formal consent was obtained. Those with any known medical illness or antibiotic therapy in the past three months, uncooperative or apprehensive, thumb sucking habit and those on antiseptic mouth wash were excluded. The study comprised of 150 individuals of both gender and sub divided into four independent groups, group 1 is the control group $(\mathrm{n}=30)$ comprised of individuals with neither nail biting habit nor orthodontic appliance use, group $2(\mathrm{n}=60)$ with only nail biting habit, group 3 $(n=30)$ with fixed orthodontic multiple bracket appliance use for a minimum of three months period and group $4(n=30)$ with a combination of chronic nail biting habit along with fixed orthodontic multiple bracket appliance use for three months or more. All the groups were mutually exclusive where participants cannot be in more than one group at any given time.

The concentrated oral rinse technique described by Samaranayake et al. (21) to detect oral Candidal species was adopted for culturing oral Enterobacteriaceae. In brief, all the subjects were provided a $10 \mathrm{ml}$ phosphate buffered saline in a sterile container and advised to rinse their mouth for a minute. The rinse is then collected in a sterile container and taken for microbiological analysis. The oral rinse was then centrifuged at 17,000 $\mathrm{g}(12,500 \mathrm{rpm})$ for ten minutes, the supernatant was discarded, and the deposit was resuspended in $1 \mathrm{ml}$ of phosphate buffered saline to yield the concentrated rinse. A full loop of concentrated rinse was inoculated in MacConkey's (M082 Hi-media Labs, India) and Blood Agar (UNSPC code- 12352200) culture media plates using Standard streak plate method. The streaked plates were incubated for 24 hours and the culture was examined for Enterobacteriaceae growth in pink and white coloured colonies. The calculation of the Colony Forming Unit per millilitre $(\mathrm{CFU} / \mathrm{ml})$ is based on the formula $\mathrm{CFU} / \mathrm{ml}$ $=$ (number of colonies multiplied by the dilution factor) divided by the volume of culture plates.

A small amount of lactose fermenting colonies was transferred aseptically with inoculating loop and smears were made. The culture was gram stained using conventional protocol for visualization under a microscope for gram-negative bacilli. Biochemical tests were performed as per manufacturer instruction for Enterobacteriaceae and sub-species specific microganisms of Escherichia coli, Enterobacter areogenes, Klebsiella and Enterobacter cloacae with the help of Biomerieux Vitek 2 Compact Automated Microbiological Analyzer10 (Fig. 1: VITEK 2 Compact system).

Descriptive statistics with frequencies and percentages were used to summarize sociodemographic and dependent variables. To test the association between nail biting, orthodontic treatment and Enterobacteriaceae a Fisher's exact test was performed. To identify the difference between groups Kruskal-Wallis test and post hoc analysis using the Dunn method (22) was performed. The $p$ value was corrected for multiple testing with Bonferroni adjustment. The Kruskal-Wallis test was pre- 


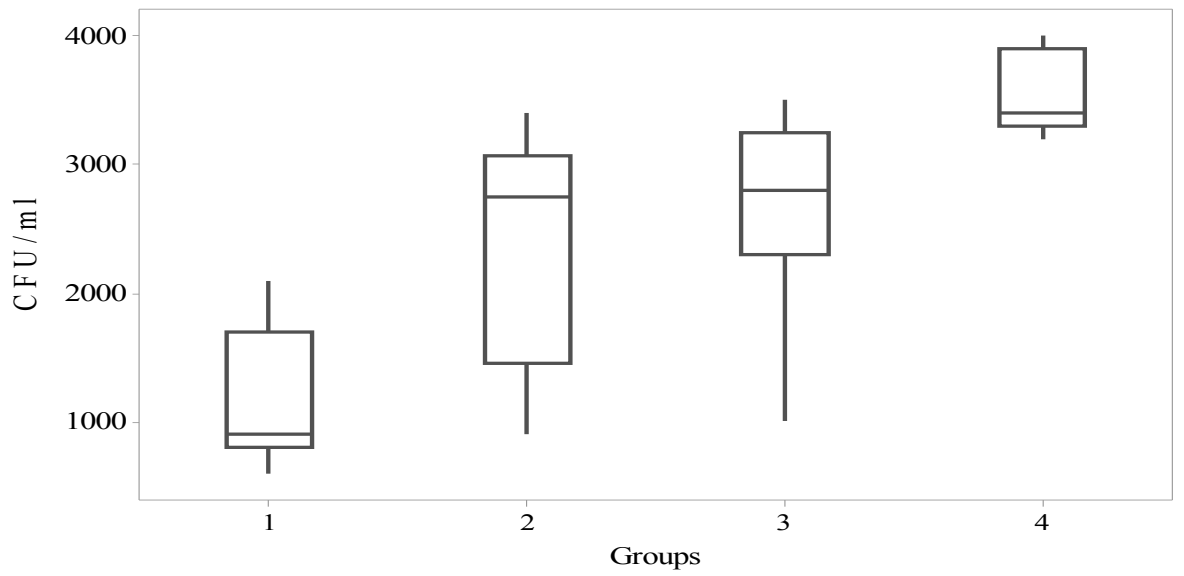

Fig. 1: CFU/ml (optional).

ferred since the assumption for normality was violated when Shapiro-Wilk's test identified $p<.05$ when Colony Forming Unit per millilitre $(\mathrm{CFU} / \mathrm{ml})$ for the four groups was assessed. The confidence interval was set at $99 \%$ and significance level at 0.01 for all the tests and the analyses was performed using Statistical Package for the Social Sciences software (SPSS for Windows, Version 24, Chicago, IL). A priori sample size was determined using GPower with the alpha set at 0.01 and power at 0.95. A large effect size $(\mathrm{f} 2=0.40)$ was used for the analysis. Based on these assumptions the minimum number of participants required for the analysis was determined as 148 .

\section{Results}

Summary of the descriptive statistics is presented in table 1 and 2. Approximately, 69\% of the participants were males. The mean age was 12.7 (SD 3.4) for males

Table 1: Descriptive statistics of participants and Enterobacteriaceae by groups.

\begin{tabular}{|l|c|c|c|c|c|c|c|c|}
\hline \multirow{2}{*}{ Groups } & Male & Female & Age & Enterobacteriaceae & \multicolumn{2}{|c|}{ CFU/ml } & Colonies \\
\cline { 2 - 8 } & $\mathrm{n}(\%)$ & $\mathrm{n}(\%)$ & Mean (SD) & $\begin{array}{c}\text { Positive } \\
\mathrm{n}(\%)\end{array}$ & $\begin{array}{c}\text { Negative } \\
\mathrm{n}(\%)\end{array}$ & Mean (SD) & Median* & Mean (SD) \\
\hline No nail biting (control) & $18(60)$ & $12(40)$ & $12(3.5)$ & $12(40)$ & $19(60)$ & $1186(525)$ & 900 & $11.8(5.2)$ \\
\hline Nail biting & $45(75)$ & $15(25)$ & $11(3.0)$ & $45(75)$ & $15(25)$ & $2416(844)$ & 2750 & $24.1(8.4)$ \\
\hline $\begin{array}{l}\text { Only Orthodontic } \\
\text { treatment }\end{array}$ & $21(70)$ & $9(30)$ & $15.3(1.4)$ & $24(80)$ & $6(20)$ & $2560(865)$ & 2800 & $25.6(8.6)$ \\
\hline $\begin{array}{l}\text { Orthodontic treatment } \\
\text { with nail biting }\end{array}$ & $20(67)$ & $10(33)$ & $14.5(1.8)$ & $27(90)$ & $3(10)$ & $3520(282)$ & 3400 & $35.2(2.8)$ \\
\hline Total/average & $104(69)$ & $46(30.7)$ & $12.8(3.2)$ & $108(72)$ & $42(28)$ & $2420(1014)$ & 2462 & $24.2(10.2)$ \\
\hline
\end{tabular}

"Kruskal Wallis; Test statistic 87.2, degree of freedom 3, Asymptomatic Sig (2-sided test) 0.001.

Table 2: Isolation frequencies of specific opportunistic bacteria.

\begin{tabular}{|l|c|c|c|c|c|}
\hline Enterobacteriaceae & \multicolumn{2}{|c|}{ Non-orthodontic } & \multicolumn{2}{c|}{ Orthodontic } & \\
\hline & Non-NB & NB & Non-NB & NB & Total \\
\hline Escherichia coli & 10 & 25 & 11 & 12 & 58 \\
\hline Enterobacter areogenes & 10 & - & - & & 10 \\
\hline Klebsiella & - & - & - & 7 & 7 \\
\hline Enterobacter cloacae & - & - & 11 & - & 11 \\
\hline
\end{tabular}


and 13 (SD 2.7) for females. No significant gender difference was observed for Enterobacteriaceae test results. Overall, Enterobacteriaceae was positive for $72 \%$ ( $\mathrm{n}=$ $108)$ of the children. Of them, $89 \%(n=96)$ were in the nail biting or orthodontic treatment group. Among those tested positive $(\mathrm{n}=108)$ for Enterobacteriaceae $88.9 \%$ $(\mathrm{n}=96)$ were in the nail biting and orthodontic treatment group compared to $11.1 \%(\mathrm{n}=12)$ in the non-nail-biting and non-orthodontic treatment group. More number of children tested positive for Enterobacteriaceae in the orthodontic treatment group (85\%) compared to $63 \%$ in the non-orthodontic treatment group and this difference was statistically significant when assessed by Fisher's exact test with a $p<0.01$. Further, a significant association for Enterobacteriaceae in those with nail biting and or orthodontic treatment group compared to those with no nail-biting habit and or not receiving orthodontic treatment as assessed by Fisher's exact test, $p<.01$. The Kruskal-Wallis test was used to identify if significant differences existed in the distribution of $\mathrm{CFU} / \mathrm{ml}$ values in the four groups of participants. Distributions of CFU $/ \mathrm{ml}$ scores were not similar for all groups, as assessed by visual inspection of the boxplot (not presented in the figure) The results of the post hoc pairwise multiple comparisons are presented in table 3 . The mean

Table 3: Post-hoc pairwise multiple comparison.

\begin{tabular}{|l|c|c|}
\hline Sample 1-sample2 & Test statistic (SE) & $\boldsymbol{p}^{*}$ value \\
\hline Group 1 - group 2 & $-46.2(9.7)$ & $<0.001$ \\
\hline Group 1 - group 3 & $-54.6(11.2)$ & $<0.001$ \\
\hline Group 1 - group 4 & $-104(11.2)$ & $<0.001$ \\
\hline Group 2 - group 3 & $-8.4(9.7)$ & $>1.00$ \\
\hline Group 2 - group 4 & $-57.8(9.7)$ & $<0.001$ \\
\hline Group 3 - group 4 & $-49.4(11.2)$ & $<0.001$ \\
\hline
\end{tabular}

rank of CFU/ml values identified statistically significant difference in five of the six pairwise multiple comparison $\chi 2(3)=87.2, p<.01$. The only instance where post hoc analysis identified no statistically significant difference was between nail biting and orthodontic treatment in isolation.

The isolated frequencies of the four Enterobacteriaceae species are presented in table 2. Overall, 39\% (58/150) of the subjects tested positive for the four organisms. This implies that 54\% (58/108) of the Enterobacteriaceae positive also tested positive for one of the four species of facultative opportunistic gram-negative baci1li. The proportion of those tested positive for the four species of the Enterobacteriaceae was the same for the orthodontic 38\% (23/58) and non-orthodontic group $38 \%(35 / 58)$. Three of the four organisms isolated tes- ted positive in the orthodontic treatment group compared to two in the non-orthodontic treatment group. All the 58 subjects tested positive for E. coli. Of them, $83 \%$ (48/58) were either in the nail-biting group or receiving orthodontic treatment. Klebsiella and E. cloacae were isolated exclusively in the orthodontic treatment group. Of them Klebsiella was isolated in those with no nail-biting habit but $E$. cloacae were present in those with nail biting habit while receiving orthodontic treatment. $E$. aerogenes was neither present in the nail-biting group nor orthodontic treatment group.

\section{Discussion}

Our study is the first to explore oral load due to nail-biting and the use of orthodontic appliances in healthy children. Our primary interest is to identify the difference in the microbial load and the number of participants in our study is more than any previously reported studies on nail biting and orthodontic treatment. Despite that they are not sufficiently powered to estimate the prevalence due to challenges with sample size and selection. Sedgley and Samaranayake (1994) reported practical difficulties in identifying the prevalence of Enterobacteriaceae due to small sample numbers, and methodology employed (13).

Our findings are consistent with previous research where Enterobacteriaceae in the oral cavity were significantly higher in nail biters compared to non-nail biters $(14,15)$. The results demonstrated higher CFU values for Enterobacteriaceae was associated with the use of fixed orthodontic appliances or the habit of nail biting. The number of people tested positive for Enterobacteriaceae was eight times higher in those with either nail biting habit or orthodontic treatment compared to the control group (non-nail biting). In the four specific microorganisms investigated $E$. coli was predominant and was observed in $39 \%(\mathrm{n}=58 / 150)$ of all the children and $58 \%$ $(n=58 / 108)$ of all those tested positive for Enterobacteriaceae. Despite the higher proportion of E. coli present in nail biters and orthodontic appliance users individual susceptibility was not observed for either of these two groups. However, Klebsiella was exclusive to those with a combination of nail biting and orthodontic treatment. Similarly, E.cloacae was observed exclusively in those receiving orthodontic treatment with no nail biting habit. Our findings are different from those observed by Kamal and Bernard (23) where E. coli and Enterobacter were isolated in children with nail biting habit and Klebsiella in the control group.

Nail biting has been associates with several dental and medical conditions. Transfer of oral microbes to the gut through the saliva is not common. A report published in Genome Biology by Segata et al. (24) stated The Human Microbiome Project established 45\% overlap between oral and stool bacteria. Nail biting increases the risk of 
accidental inoculation of environmental organism into the oral cavity and the risk is particularly high in children do not wash their hands properly. The relatively immature immune system does not offer adequate protection as in adults and food-borne environmental pathogens can gain entry to the oral cavity when good hand hygiene is unlikely to be optimal. This makes them particularly vulnerable for Enterobacteriaceae infection that is becoming increasingly ineffective to routine antibiotics Most of the literature on Enterobacteriaceae have focussed on antimicrobial resistance. Commonly used antimicrobials have become ineffective with the emergence of several multi drug resistance Enterobacteriaceae, globally (25-27). The extended spectrum beta lactamase producing Enterobacteriaceae is the most common cause of this public health concern. E. coli, Klebsiella pneumoniae and Enterobacter species are notoriously known for their pathogenic potential and have been consistently demonstrated the highest prevalence of betalactamase production in children $(28,29)$. The extended-spectrum $\beta$-lactamase-associated infections are reported to have four times higher mortality and enduring bacteraemia compared to those without this infection (30). A systematic review and meta-analysis of multi drug resistance E. coli in asymptomatic children identified a pooled prevalence of $28.6 \%$ to $37.7 \%$ for Organisation for Economic Cooperation and Development (OECD) countries and $67.2 \%$ to $81.3 \%$ for non-OECD countries(27). South-East Asia and Western Pacific regions hold 4.3 of the 7.7 billion world population and that includes the regional heavy weight China and India where antimicrobial resistance for extended-spectrum $\beta$-lactamase-producing bacteria in children is particularly high (28).

\section{Conclusions}

Nail biting or the use of fixed orthodontic appliances is associated with higher incidence of Enterobacteriaceae in the oral cavity that can influence the quantity and quality of oral microbiota that have important implication in systemic diseases and conditions. Oral health professionals must ensure that habitual nail biting is recorded in the patient medical and dental history and every effort should be made to ensure the habit is discouraged. Children and accompanying parents should be informed of the adverse consequence in every follow-up visit. The importance of maintaining good hand and oral hygiene should be emphasized at every available opportunity during orthodontic treatment.

\section{References}

1. Halteh P, Scher RK, Lipner SR. Onychophagia: A nail-biting conundrum for physicians. The Journal of dermatological treatment. 2017;28:166-72.

2. Garde J, Suryavanshi RK, Jawale BA, Deshmukh V, Dadhe DP, Suryavanshi MK. An epidemiological study to know the prevalence of deleterious oral habits among 6 to 12 year old children. Journal of international oral health: JIOH. 2014;6:39.
3. Jabr FI. Severe nail deformity. Nail biting may cause multiple adverse conditions. Postgrad Med. 2005;118:37-8, 42.

4. Bienvenu OJ, Samuels JF, Riddle MA, Hoehn-Saric R, Liang KY, Cullen BA, et al. The relationship of obsessive-compulsive disorder to possible spectrum disorders: results from a family study. Biol Psychiatry. 2000;48:287-93.

5. Pastro JDV, Nogueira ACA, Salvatore de Freitas KM, Valarelli FP, Cancado RH, de Oliveira RCG, et al. Factors Associated to Apical Root Resorption after Orthodontic Treatment. The open dentistry journal. 2018;12:331-9.

6. Emodi-Perlman A, Eli I, Friedman-Rubin P, Goldsmith C, Reiter $\mathrm{S}$, Winocur E. Bruxism, oral parafunctions, anamnestic and clinical findings of temporomandibular disorders in children. J Oral Rehabil. 2012;39:126-35.

7. Castelo PM, Gaviao MB, Pereira LJ, Bonjardim LR. Relationship between oral parafunctional/nutritive sucking habits and temporomandibular joint dysfunction in primary dentition. Int J Paediatr Dent. 2005;15:29-36.

8. Farsi N, Alamoudi N, Feteih R, El-Kateb M. Association between temporo mandibular disorders and oral parafunctions in Saudi children. Odontostomatol Trop. 2004;27:9-14.

9. Lavigne G, Kato T. Usual and unusual orofacial motor activities associated with tooth wear. Int J Prosthodont. 2005;18:291-2.

10. Bello J, Nunez FA, Gonzalez OM, Fernandez R, Almirall P, Escobedo AA. Risk factors for Giardia infection among hospitalized children in Cuba. Ann Trop Med Parasitol. 2011;105:57-64.

11. Wendt S, Trawinski H, Schubert S, Rodloff AC, Mossner J, Lubbert C. The Diagnosis and Treatment of Pinworm Infection. Deutsches Arzteblatt international. 2019;116:213-9.

12. Siqueira JF Jr, Rocas IN. The Oral Microbiota in Health and Disease: An Overview of Molecular Findings. Methods Mol Biol. 2017;1537:127-38.

13. Sedgley CM, Samaranayake LP. Oral and oropharyngeal prevalence of Enterobacteriaceae in humans: a review. J Oral Pathol Med. 1994;23:104-13.

14. Baydas B, Uslu H, Yavuz I, Ceylan I, Dagsuyu IM. Effect of a chronic nail-biting habit on the oral carriage of Enterobacteriaceae. Oral Microbiol Immunol. 2007;22:1-4.

15. Reddy S, Sanjai K, Kumaraswamy J, Papaiah L, Jeevan M. Oral carriage of enterobacteriaceae among school children with chronic nail-biting habit. J Oral Maxillofac Pathol. 2013;17:163-8.

16. Kitada K, De Toledo A, Oho T. Increase in detectable opportunistic bacteria in the oral cavity of orthodontic patients. Int J Dent Hyg. 2009;7:121-5.

17. Pathak AK, Sharma DS. Biofilm associated microorganisms on removable oral orthodontic appliances in children in the mixed dentition. J Clin Pediatr Dent. 2013;37:335-9.

18. Jia G, Zhi A, Lai PFH, Wang G, Xia Y, Xiong Z, et al. The oral microbiota - a mechanistic role for systemic diseases. Br Dent J. 2018;224:447-55

19. Olsen I, Yamazaki K. Can oral bacteria affect the microbiome of the gut? J Oral Microbiol. 2019;11:1586422.

20 . Vogel LD. When children put their fingers in their mouths. Should parents and dentists care? NY State Dent J. 1998;64:48-53.

21. Samaranayake LP, MacFarlane TW, Lamey PJ, Ferguson MM. A comparison of oral rinse and imprint sampling techniques for the detection of yeast, coliform and Staphylococcus aureus carriage in the oral cavity. J Oral Pathol. 1986;15:386-8.

22. Dunn OJ. Multiple comparisons using rank sums. Technometrics. 1964;6:241-52.

23. Kamal FG, Bernard RA. Influence of nail biting and finger sucking habits on the oral carriage of Enterobacteriaceae. Contemp Clin Dent. 2015;6:211-4.

24. Segata N, Haake SK, Mannon P, Lemon KP, Waldron L, Gevers $\mathrm{D}$, et al. Composition of the adult digestive tract bacterial microbiome based on seven mouth surfaces, tonsils, throat and stool samples. Genome Biol. 2012;13:R42.

25. Meropol SB, Haupt AA, Debanne SM. Incidence and Outcomes of Infections Caused by Multidrug-Resistant Enterobacteriaceae in Chil- 
dren, 2007-2015. Journal of the Pediatric Infectious Diseases Society. 2018;7:36-45.

26. Logan LK, Renschler JP, Gandra S, Weinstein RA, Laxminarayan R, Program PE, et al. Carbapenem-resistant enterobacteriaceae in children, United States, 1999-2012. Emerg Infect Dis. 2015;21:2014. 27. Bryce A, Costelloe C, Hawcroft C, Wootton M, Hay AD. Faecal carriage of antibiotic resistant Escherichia coli in asymptomatic children and associations with primary care antibiotic prescribing: a systematic review and meta-analysis. BMC Infect Dis. 2016;16:359.

28. Badal RE, Bouchillon SK, Lob SH, Hackel MA, Hawser SP, Hoban DJ. Etiology, extended-spectrum beta-lactamase rates and antimicrobial susceptibility of gram-negative bacilli causing intra-abdominal infections in patients in general pediatric and pediatric intensive care units--global data from the Study for Monitoring Antimicrobial Resistance Trends 2008 to 2010. Pediatr Infect Dis J. 2013;32:636-40.

29. Livermore DM, Woodford N. The beta-lactamase threat in Enterobacteriaceae, Pseudomonas and Acinetobacter. Trends Microbiol. 2006;14:413-20.

30. Hu YJ, Ogyu A, Cowling BJ, Fukuda K, Pang HH. Available evidence of antibiotic resistance from extended-spectrum $\beta$-lactamase-producing Enterobacteriaceae in paediatric patients in 20 countries: a systematic review and meta-analysis. Bull World Health Organ. 2019;97:486.

\section{Conflict of interest}

The authors have declared that no conflict of interest exist. 